\title{
Gamma Knife surgery for incidental cerebral arteriovenous malformations
}

\author{
Clinical article
}

\author{
Chun-Po Yen, M.D., ${ }^{1}$ Dale Ding, M.D., ${ }^{1}$ Ching-Hsiao Cheng, M.D., ${ }^{2}$ \\ Robert M. Starke, M.D., M.Sc., ${ }^{1}$ Mark Shaffrey, M.D., ${ }^{1}$ \\ and Jason Sheehan, M.D., Ph.D., ${ }^{1,3}$
}

${ }^{1}$ Department of Neurological Surgery, University of Virginia, Charlottesville, Virginia; ${ }^{2}$ Department of Neurosurgery, Kaohsiung Chang Gung Memorial Hospital and Chang Gung University College of Medicine, Kaohsiung, Taiwan; and ${ }^{3}$ Department of Radiation Oncology, University of Virginia, Charlottesville, Virginia

\begin{abstract}
Object. A relatively benign natural course of unruptured cerebral arteriovenous malformations (AVMs) has recently been recognized, and the decision to treat incidentally found AVMs has been questioned. This study aims to evaluate the long-term imaging and clinical outcomes of patients with asymptomatic, incidentally discovered AVMs treated with Gamma Knife surgery (GKS).

Methods. Thirty-one patients, each with an incidentally diagnosed AVM, underwent GKS between 1989 and 2009. The nidus volumes ranged from 0.3 to $11.1 \mathrm{~cm}^{3}$ (median $3.2 \mathrm{~cm}^{3}$ ). A margin dose between 15 and $26 \mathrm{~Gy}$ (median $20 \mathrm{~Gy}$ ) was used to treat the AVMs. Four patients underwent repeat GKS for still-patent AVM residuals after the initial GKS procedure. Clinical follow-up ranged from 24 to 196 months, with a mean of 78 months (median 51 months) after the initial GKS.

Results. Following GKS, 19 patients (61.3\%) had a total AVM obliteration on angiography. In 7 patients $(22.6 \%)$, no flow voids were observed on MRI but angiographic confirmation was not available. In 5 patients (16.1\%), the AVMs remained patent. A small nidus volume was significantly associated with increased AVM obliteration rate. Thirteen patients (41.9\%) developed radiation-induced imaging changes: 11 were asymptomatic (35.5\%), 1 had only headache $(3.2 \%)$, and 1 developed seizure and neurological deficits $(3.2 \%)$. Two patients each had 1 hemorrhage during the latency period (116.5 risk years), yielding an annual hemorrhage rate of $1.7 \%$ before AVM obliteration.

Conclusions. The decision to treat asymptomatic AVMs, and if so, which treatment approach to use, remain the subject of debate. GKS as a minimally invasive procedure appears to achieve a reasonable outcome with low procedure-related morbidity. In those patients with incidental AVMs, the benefits as well as the risks of radiosurgical intervention will only be fully defined with long-term follow-up.
\end{abstract}

(http://thejns.org/doi/abs/10.3171/2014.7.JNS131397)

\section{KEY WORDS • arteriovenous malformations • asymptomatic • hemorrhage • Gamma Knife surgery $\quad \bullet \quad$ vascular disorders $\quad \bullet \quad$ stereotactic radiosurgery}

$\mathrm{A}$ LTHOUGH treatment for cerebral arteriovenous malformations (AVMs) might be directed toward reducing seizure activities, stopping "vascular steal," or alleviating neurological deficits caused by mass effect, the main goal of any intervention for AVMs is complete obliteration of the nidus, thereby eliminating subsequent hemorrhage risk. The traditionally quoted $2 \%-4 \%$ annual hemorrhage rates of AVMs have led to a general philosophy among neurosurgeons that all AVMs should be aggressively treated given the high accumu-

Abbreviations used in this paper: ARUBA = A Randomized Trial of Unruptured Brain Arteriovenous Malformations; AVM = arteriovenous malformation; GKS = Gamma Knife surgery. lated lifetime hemorrhage risk in the relatively young patient population harboring these lesions. With recent studies demonstrating that the natural history of unruptured AVMs may differ from their ruptured counterparts, controversy has arisen over the proper management of unruptured AVMs. Furthermore, for incidentally diagnosed AVMs, which are entirely asymptomatic, any intervention is completely prophylactic and therefore only justifiable if the risks of morbidity and mortality associated with treatment are not greater than those of the natural course of AVMs. Thus, whether an incidental AVM

This article contains some figures that are displayed in color online but in black-and-white in the print edition. 
should be treated remains controversial. Given its minimal invasiveness and generally favorable benefit-to-risk profile, Gamma Knife surgery (GKS) has emerged as a popular treatment tool for AVMs without immediate risk of hemorrhage. The aim of the current study is to evaluate the obliteration rate of incidental AVMs following GKS, the risk of hemorrhage after GKS, and the morbidity and mortality associated with this treatment modality.

\section{Methods}

\section{Patient Population}

Between May 1989 and September 2009, a total of 1400 patients with AVMs were treated with GKS at the University of Virginia. Among these 1400 patients, $35(2.5 \%)$ had AVMs discovered incidentally. Four patients with follow-up shorter than 2 years after GKS were excluded, leaving 31 patients for analysis in this study. There were 20 males and 11 females with a median age of 39.6 years (mean 38.6 years, range 6-71 years; Table 1). Five patients were younger than 18 years old at the time of GKS. Three patients volunteered during imaging research and were diagnosed with AVMs. Nine patients were found to have AVMs on images obtained during the evaluation for head injury. None of these patients had seizures leading to the accidents. The AVMs of the other 19 patients were found during the workup for other medical diseases: 3 for multiple sclerosis, 2 for cervical degeneration, 2 for facial angiomas, 2 for coronary artery diseases, 3 for endocrine dysfunctions, and 1 each for carotid stenosis, Tourette syndrome, depression, autism, eye surgery, otosclerosis, and toxic epidermal necrolysis.

None of the patients underwent microsurgical resection before GKS. Five patients (16.1\%) underwent embolization prior to GKS to reduce the volume of the nidus. The median duration between the diagnosis of the AVMs and GKS was 7 months (mean 36 months, range 1 month to 36 years).

\section{AVM Characteristics}

The locations of AVM nidi were lobar in 20 (4 frontal, 4 temporal, 5 parietal, and 7 occipital), basal ganglia in 3 , corpus callosum in 2 , brainstem in 3 , cerebellum in 1 , and sylvian fissure in 2 . The nidus volumes ranged from 0.3 to $11.1 \mathrm{~cm}^{3}$ (median $3.2 \mathrm{~cm}^{3}$, mean $3.6 \mathrm{~cm}^{3}$ ). Seventeen nidi had only superficial venous drainage, 6 had only deep venous drainage, and 8 had both. The SpetzlerMartin grading at the time of initial GKS was Grade I in 4 patients, Grade II in 13 patients, Grade III in 12 patients, and Grade IV in 2 patients (Table 1). The median "modified radiosurgery-based AVM scale" score as proposed by Pollock and Flickinger was 1.24 (medium 1.23, range $0.23-1.91){ }^{11}$

Four patients underwent repeat GKS for still-patent AVM residuals performed at a mean of 6.8 years $(5.0,5.6$, 12.1 , and 4.5 years) after the initial GKS. The mean nidus volume was $1.9 \mathrm{~cm}^{3}\left(0.6,0.8,1.8\right.$, and $\left.4.2 \mathrm{~cm}^{3}\right)$.

\section{GKS Procedure}

The details of GKS procedures at our institution have
TABLE 1: Patient demographics, AVM characteristics, and GKS parameters

\begin{tabular}{|c|c|}
\hline Variable & Value \\
\hline \multicolumn{2}{|l|}{ patient demographics $(n=31)$} \\
\hline \multicolumn{2}{|l|}{$\operatorname{sex}(\%)$} \\
\hline male & $20(64.5)$ \\
\hline female & $11(35.5)$ \\
\hline \multicolumn{2}{|l|}{ age at initial GKS (yrs) } \\
\hline median & 39.6 \\
\hline mean & 38.6 \\
\hline range & $6-71$ \\
\hline \multicolumn{2}{|l|}{ reasons AVM found (\%) } \\
\hline volunteer in imaging research & $3(9.7)$ \\
\hline imaging for head injury workup & $9(29.0)$ \\
\hline imaging for other medical diseases & $19(61.3)$ \\
\hline \multicolumn{2}{|l|}{ interval between diagnosis \& GKS (mos) } \\
\hline median & 7 \\
\hline mean & 36 \\
\hline range & $1-432$ \\
\hline \multicolumn{2}{|l|}{ AVM characteristics ( $n=31$ ) } \\
\hline \multicolumn{2}{|l|}{ volume of the nidus $\left(\mathrm{cm}^{3}\right)$} \\
\hline median & 3.2 \\
\hline mean & 3.6 \\
\hline range & $0.3-11.1$ \\
\hline \multicolumn{2}{|l|}{ locations (\%) } \\
\hline lobar & $20(64.5)$ \\
\hline frontal/temporal/parietal/occipital & $4 / 4 / 5 / 7$ \\
\hline basal ganglia & $3(9.7)$ \\
\hline corpus callosum & $2(6.5)$ \\
\hline brainstem & $3(9.7)$ \\
\hline cerebellum & $1(3.2)$ \\
\hline insula/sylvian fissure & $2(6.5)$ \\
\hline \multicolumn{2}{|l|}{ Spetzler-Martin Grade (\%) } \\
\hline I & $4(12.9)$ \\
\hline$\|$ & $13(41.9)$ \\
\hline III & $12(38.7)$ \\
\hline IV & $2(6.5)$ \\
\hline \multicolumn{2}{|l|}{ initial treatment parameters $(n=31)$} \\
\hline \multicolumn{2}{|l|}{ margin dose (Gy) } \\
\hline median & 20 \\
\hline mean & 20.8 \\
\hline range & $15-26$ \\
\hline \multicolumn{2}{|l|}{ maximum dose (Gy) } \\
\hline median & 40 \\
\hline mean & 40.5 \\
\hline range & $30-52$ \\
\hline \multicolumn{2}{|l|}{ isodose line (\%) } \\
\hline median & 50 \\
\hline mean & 51.6 \\
\hline range & $50-70$ \\
\hline
\end{tabular}

(continued) 
TABLE 1: Patient demographics, AVM characteristics, and GKS parameters (continued)

\begin{tabular}{ll}
\hline \multicolumn{1}{c}{ Variable } & Value \\
\hline initial treatment parameters $(n=31)$ (continued) & \\
isocenters & \\
median & 2 \\
mean & 3.1 \\
range & $1-12$ \\
\hline
\end{tabular}

been previously described. ${ }^{4,13,16}$ The Leksell Gamma Unit Model U was used until July 2001 when the $C$ model (Elekta Instruments, Inc.) replaced it. Stereotactic biplane angiography was the only imaging modality available for nidus definition and dose planning until 1991, when stereotactic MRI was routinely used as a supplement to enhance the spatial accuracy of angiography for treatment planning. The Kula software was used for dose planning from 1989 to June 1994 and then was replaced with Gamma Plan software.

\section{Treatment Parameters}

The treatment parameters at the initial GKS were as follows (Table 1): median margin dose 20 Gy (mean 20.8 Gy, range 15-26 Gy); median maximum dose 40 Gy (mean $40.5 \mathrm{~Gy}$, range $30-52 \mathrm{~Gy}$ ); median isodose line $50 \%$ (mean $52 \%$, range 50\%-70\%); and median number of isocenters 2 (mean 3.1, range 1-12). The treatment parameters of the second GKS were: median and mean margin dose 23 Gy $(25,23,23$, and $21 \mathrm{~Gy})$; median and mean maximum dose 46 Gy $(50,46,46$, and $42 \mathrm{~Gy})$; isodose line $50 \%$ in all patients; and median number of isocenters 3 (mean 3.3, range 1-6).

\section{Clinical and Imaging Follow-Up After GKS}

The patients treated earlier were subjected to a vigorous protocol of yearly angiograms. Later, with the introduction of MRI, angiography was not performed until the nidus was no longer visible on MRI performed at 6-month intervals for the first 2 years, then yearly afterward. Additional CT or MRI was performed if the patient declined neurologically. When no more flow voids could be detected on MRI, suggesting complete AVM occlusion, angiography was performed to confirm obliteration of the nidus. Thereafter, patients were advised to undergo further MRI every 5 years to rule out delayed adverse effects. The median MRI follow-up was 36.8 months (mean 57.5 months, range $24-163$ months). Two patients did not undergo follow-up angiography due to patent nidi on MRI. In 7 patients, either the patients themselves or their families refused to have an angiogram in spite of no flow voids visible on MRI. Of the remaining 22 patients, the median angiographic follow-up was 43.5 months (mean 54 months, range 24-173 months).

Follow-up data were obtained from the patients and referring physicians. Clinical and imaging responses as well as GKS-related complications were evaluated. Clinical follow-up was $2-5$ years in 16 patients $(51.6 \%), 5-10$ years in 7 patients (22.6\%), 10-15 years in 5 patients $(16.1 \%)$, and greater than 15 years in 3 patients $(9.7 \%)$. The clinical follow-up ranged from 24 to 196 months (median 51 months, mean 78 months).

\section{Statistical Analysis}

All statistical analyses were performed using the statistical software package SPSS (version 20, IBM). Time to obliteration was calculated using the Kaplan-Meier method. The time of obliteration was based on the date of MRI when the AVMs were no longer visible because angiography was only prompted after MRI no longer visualized the nidi. Of note, obliteration of the AVM nidi might occur earlier as the MRI was performed only at 6- or 12-month intervals. The true time to obliteration probably fell between the dates of last MRI when the nidi were still visible and the dates when the nidi were interpreted as obliterated on MRI. Univariate and multivariate analyses were computed using Cox proportional hazards models to evaluate predictive factors of nidus obliteration. Relevant factors examined include sex, age, history of preradiosurgical embolization, AVM volume, location of nidi (superficial vs deep location), draining veins (superficial vs deep, multiple vs single), margin dose, maximum dose, isodose, number of isocenters, modified radiosurgery-based AVM score,,$^{11}$ and presence of radiation-induced imaging changes after GKS. ${ }^{14}$ Univariate and multivariate logistic regression were used to analyze factors potentially affecting the occurrence of radiation-induced imaging changes. A p value of less than 0.05 was used as the limit for statistical significance. The postradiosurgical hemorrhage rate was calculated by dividing the number of hemorrhages after radiosurgery by total risk years, which is the sum of either time after radiosurgery to obliteration or, in patients without total obliteration, to last clinical follow-up.

\section{Results}

\section{Imaging Outcome}

Following a single GKS procedure, 8 patients (25.8\%) still had a residual nidus shown on MRI or angiography. In 4 patients (12.9\%), the last MRI examination revealed absence of flow voids, but the patients refused to undergo angiography to confirm the obliteration of nidi. In 19 patients $(61.3 \%)$, obliteration of the nidus was confirmed on follow-up angiography (Figs. 1 and 2). In 4 patients harboring residual AVMs who underwent repeat GKS, there were no flow voids on MRI in 3 of these patients. In 1 patient, the nidus remained patent at last follow-up (Fig. 2).

Overall, following 1 GKS procedure or repeat GKS, a total AVM obliteration was confirmed on angiography in 19 patients $(61.3 \%)$. Five patients $(16.1 \%)$ continued to have patent residual nidi (visualized on MRI in 2 patients and on angiography and MRI in 3 patients). In 7 patients $(22.6 \%)$, obliteration of the nidi was assumed by the fact that there were no flow voids visible on MRI (Fig. 2). The median time to obliteration based on MRI was 30.4 months. The actuarial angiographic or MRI obliteration rate was $55 \%$ and $78 \%$ at 3 and 5 years following GKS, respectively (Fig. 3). 


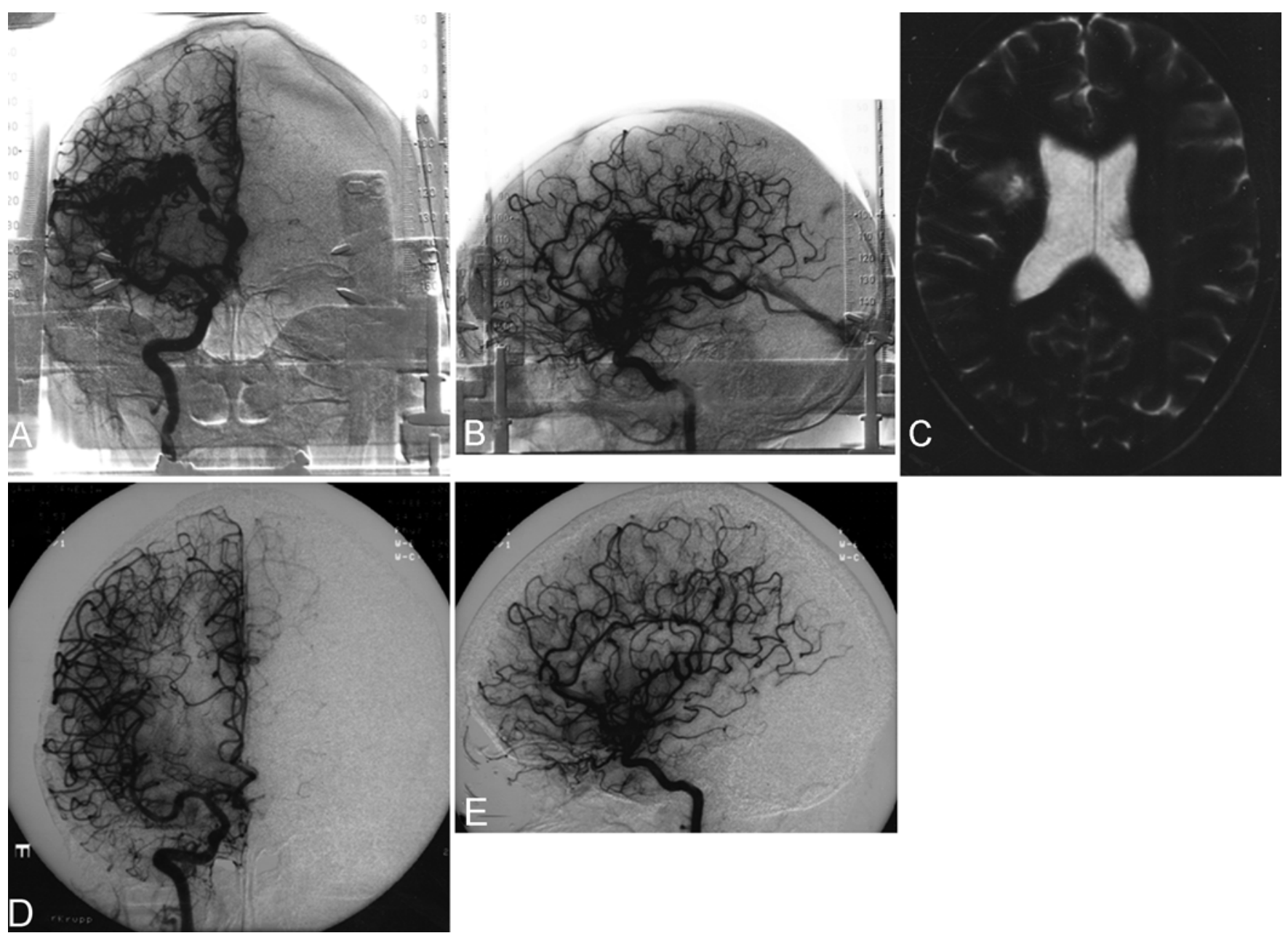

FIG. 1. Images obtained in a 40-year-old woman with an incidental AVM involving the right caudate nucleus. On frontal (A) and lateral (B) projection angiograms, the nidus measured $25 \times 19 \times 13 \mathrm{~mm}$. The patient underwent GKS with a margin dose of $23 \mathrm{~Gy}$. The nidus decreased in size on MRI at the 6-month follow-up. Also noted was increased T2 signal surrounding the nidus on axial T2-weighted MRI (C). The AVM was obliterated 3 years following radiosurgery, as noted on frontal (D) and lateral (E) projection angiograms.

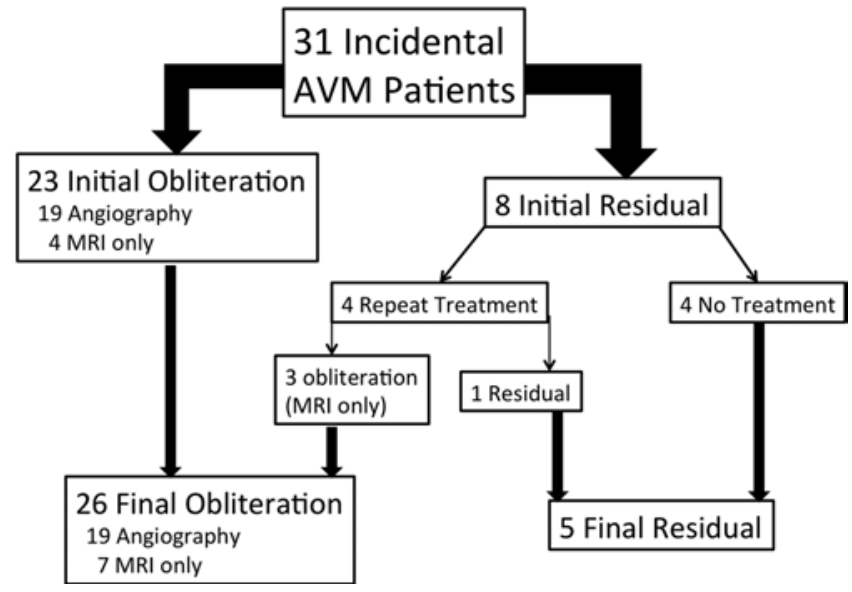

FIG. 2. Outcomes of 31 asymptomatic AVMs treated using GKS.

\section{Factors Related to Nidus Obliteration}

Nidi obliterated after the initial GKS on angiography were compared with those that were still patent (including patients with patent nidi as shown on either MRI or angiography). Univariate analysis (Table 2) demonstrated that high margin dose $(\mathrm{p}=0.042)$, high maximum dose $(\mathrm{p}=0.035)$, deep draining vein $(\mathrm{p}=0.042)$ and small nidus volume (0.015) were associated with increased AVM obliteration rate. Sex $(\mathrm{p}=0.778)$, age $(\mathrm{p}=0.619)$, prior embolization $(p=0.101)$, locations of nidi $(p=0.726)$, radiosurgery-based AVM scores $(p=0.226)$, single draining vein $(p=0.779)$, isodose line $(p=0.635)$, number of isocenters $(\mathrm{p}=0.722)$, and presence of radiation-induced imaging changes $(\mathrm{p}=0.969)$ were not related to nidus obliteration. In multivariate analysis, only small nidus volume $(\mathrm{p}=0.047)$ was significantly associated with increased rate of AVM obliteration (Table 2).

\section{Hemorrhage After GKS}

Following GKS, 2 patients each had a single hemorrhage during the latency period during a total of 116.5 risk 


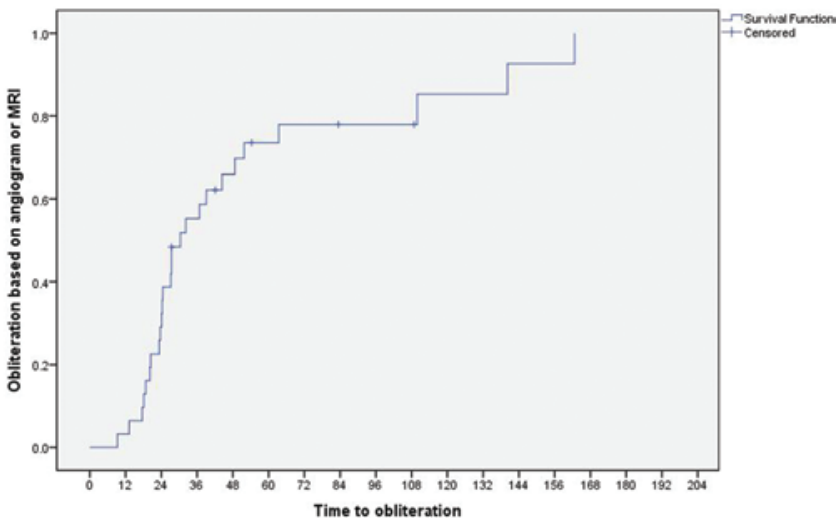

FIG. 3. Kaplan-Meier curve depicting the AVM obliteration rate following GKS in the present series.

years (assuming patients with completely obliterated AVMs were no longer at risk for hemorrhage), yielding an annual postradiosurgery hemorrhagic rate of $1.7 \%$. The first patient had a symptomatic hemorrhage from a basal ganglion AVM 24 months after GKS, and his nidus remained patent at 30 months' follow-up. The patient remained hemiparetic at the last follow-up. The second patient suffered a hemorrhage from her corpus callosum AVM 54 months after GKS. She underwent repeat GKS 12 years after the initial procedure, and her AVM was no longer visible on MRI 18 months following repeat GKS. The patient had left hemiparesis following hemorrhage, which resolved completely at the last clinical follow-up. Of the remaining 29 patients, the clinical condition of 2 patients deteriorated due to progression of their medical comorbidities (1 with multiple sclerosis, 1 with toxic epidermal necrolysis). The AVM nidi were obliterated in both patients, 1 based on MRI only, 1 confirmed by angiography. Of note, none of the 4 patients excluded from the present study due to follow-up shorter than 2 years had a hemorrhage following GKS (follow-up of $6,12,18$, and 18 months).

\section{Morbidities Associated With GKS}

Radiation-induced imaging changes visualized as increased $\mathrm{T} 2$ signal surrounding the nidi ${ }^{14}$ were observed in 13 patients (42\%). The mean interval following GKS until identification of the imaging changes was 13.7 months (median 8.7 months, range 6-60 months). The changes resolved after a mean of 23 months (median 22.1 months, range 10-44 months). On multivariate logistic regression, AVM nidi with deep draining veins $(\mathrm{p}=0.027)$ were more likely to present with radiation-induced imaging changes (Table 3).

Eleven patients (35.5\%) with imaging findings of radiation-induced imaging changes were asymptomatic. One patient presented with headache only, and the other presented with new onset seizures and mild hemiparesis. Both patients' symptoms completely resolved after a short course of steroid treatment.

\section{Discussion}

The most devastating complication of cerebral AVMs is rupture of the nidi resulting in intracerebral hemorrhage. With increasing long-term natural history studies available, there is a tendency to dichotomize the AVMs into ruptured or unruptured ones, as unruptured AVMs appear to have a more indolent clinical course., ${ }^{1,5}$ The overall AVM rupture rates have been documented in the range of $2 \%-4 \%{ }^{3,7,10,12}$ Literature reporting the AVM hemorrhage rates, however, encompasses AVM patients with or without a prior hemorrhage. The natural history of unruptured AVMs remains poorly defined. Brown et al. published a study including only unruptured AVMs. ${ }^{2}$ In this case series, a group of 168 patients with unruptured AVMs was followed for a mean of 8.2 years and a $2.2 \%$ annual hemorrhage rate was reported. Recent natural history data suggest that the annual hemorrhage rate of unruptured AVMs may be as low as $1 \% .^{1,5}$ The lower

TABLE 2: Univariate and multivariate analysis of possible predictive factors for AVM obliteration on angiography or MRI following GKS

\begin{tabular}{|c|c|c|c|c|c|c|}
\hline \multirow[b]{2}{*}{ Factor } & \multicolumn{3}{|c|}{ Univariate } & \multicolumn{3}{|c|}{ Multivariate } \\
\hline & $\mathrm{HR}$ & $95 \% \mathrm{Cl}$ & $\mathrm{p}$ Value & $\mathrm{HR}$ & $95 \% \mathrm{Cl}$ & p Value \\
\hline female & 1.122 & $0.503-2.505$ & 0.778 & & & \\
\hline age at GKS & 1.006 & $0.983-1.030$ & 0.619 & & & \\
\hline pre-GKS embolization & 0.186 & $0.025-1.386$ & 0.101 & & & \\
\hline AVM volume & 0.753 & $0.598-0.947$ & $0.015^{*}$ & 0.786 & $0.580-0.968$ & $0.047^{*}$ \\
\hline deep location & 1.172 & $0.483-2.845$ & 0.726 & & & \\
\hline deep draining vein & 0.740 & $0.553-0.989$ & $0.042^{*}$ & 2.239 & $0.780-6.422$ & 0.134 \\
\hline single draining vein & 1.020 & $0.890-1.167$ & 0.779 & & & \\
\hline margin dose & 1.167 & $1.006-1.355$ & $0.042^{*}$ & 1.063 & $0.770-1.470$ & 0.709 \\
\hline maximum dose & 1.090 & $1.006-1.181$ & $0.035^{*}$ & 0.997 & $0.831-1.196$ & 0.974 \\
\hline isodose line & 1.022 & $0.934-1.119$ & 0.635 & & & \\
\hline isocenters & 0.963 & $0.782-1.186$ & 0.722 & & & \\
\hline radiation-induced changes & 1.016 & $0.460-2.244$ & 0.969 & & & \\
\hline radiosurgery-based AVM score & 0.681 & $0.366-1.268$ & 0.226 & & & \\
\hline
\end{tabular}

* Statistically significant value $(p<0.05)$. 
TABLE 3: Multivariate logistic regression analysis of risk factors for presence or absence of radiation-induced changes

\begin{tabular}{lcll}
\hline \multicolumn{1}{c}{ Variable } & OR & \multicolumn{1}{c}{$95 \% \mathrm{Cl}$} & $\mathrm{p}$ Value \\
\hline female & 0.698 & $0.154-3.167$ & 0.642 \\
age at GKS & 0.985 & $0.941-1.030$ & 0.496 \\
pre-GKS embolization & 0.292 & $0.029-2.977$ & 0.299 \\
AVM volume & 1.171 & $0.831-1.649$ & 0.367 \\
deep location & 3.000 & $0.632-14.232$ & 0.167 \\
deep draining vein & 1.802 & $1.069-3.036$ & $0.027^{*}$ \\
single draining vein & 1.058 & $0.837-1.337$ & 0.636 \\
margin dose & 0.884 & $0.678-1.154$ & 0.365 \\
maximum dose & 0.947 & $0.829-1.082$ & 0.425 \\
isodose & 0.993 & $0.864-1.142$ & 0.922 \\
isocenters & 1.005 & $0.723-1.395$ & 0.978 \\
radiosurgery-based AVM score & 2.788 & $0.580-13.409$ & 0.201 \\
\hline
\end{tabular}

* Statistically significant value $(p<0.05)$.

rate of hemorrhage in unruptured AVMs has caused some within the cerebrovascular community to call for expectant management of these patients. Study designers of A Randomized Trial of Unruptured Brain Arteriovenous Malformations (ARUBA) have questioned the benefits of various treatments directed to obliterate or extirpate unruptured AVMs, given the low hemorrhage risk and the potential adverse events of surgery, embolization, and radiation therapy, including radiosurgery. Critics of this study have raised concerns that the cumulative lifetime hemorrhage risk of unruptured AVMs remains significant even with a $1 \%$ annual hemorrhage rate, given that most AVMs manifest themselves in adolescents and the proposed 5-year follow-up end point in the study design is not adequate to evaluate the long-term outcome of unruptured AVMs. ${ }^{9}$ The primary end point of the ARUBA study is death or stroke, and the short-term benefits of AVM treatment such as alleviation of headaches, epilepsy, and steal-induced ischemia are not addressed. Additionally, the inclusion criteria of "treatable" patients and exclusion of patients who clearly would benefit from intervention or observation that would bias the randomization are questioned. Also, the study was neither designed nor powered to discern differences among the 3 principle treatment modalities of resection, embolization, and radiosurgery. Nevertheless, the National Institute of Neurological Disorders and Stroke has recently halted enrollment in the ARUBA study due to higher than expected event rates in the interventional group, because an interim analysis of data after 33 months of follow-up from 224 of the target 400 participants showed that the event rate in the intervention group was more than 3 times higher than in the medical management group. The Data Safety and Monitoring Board of the study did recommend extended follow-up to determine whether the disparity in event rates will persist over time. It remains unclear to what extent the event rate factored into severity of sequelae. Certainly, all medical events are not of equal concern to patients with AVMs as they relate to neurological function and life expectancy.
Historically, microsurgical resection remains the gold standard of treatment for ruptured AVMs. The risk of hemorrhage is eliminated immediately with a complete extirpation of the AVM nidus. Gamma Knife surgery, on the other hand, induces intimal proliferation, smooth muscle migration, and eventually vascular channel occlusion, a process that takes 2 to 3 years before complete obliteration of the AVM nidus. Whether these radiosurgery-induced microscopic changes alter the risk of hemorrhage before complete obliteration of AVMs is uncertain. Recent studies demonstrate that the thickening of vessels might reduce tension of the vascular wall and likely protects the nidus from rupture. ${ }^{8}$ Our recent study of 1204 patients with AVMs treated using GKS demonstrated that the annual hemorrhage rate was reduced from $6.6 \%$ to $2.5 \%$ during the latency period..$^{15}$ The annual hemorrhage rate in the "high risk" subgroup of 605 patients who initially presented with a hemorrhage was reduced from $10.4 \%$ to $2.8 \%$. In the "low risk" subgroup of 599 patients who never had experienced a hemorrhage, the annual hemorrhage rate was reduced from $3.9 \%$ to $2.2 \%$. Among the $68 \%$ of patients who obtained a total obliteration of their AVMs based on angiography or MRI with a mean follow-up of 80 months after GKS, none experienced a hemorrhage once the AVMs were concluded to be obliterated. ${ }^{13}$

In the contemporary era with tomographic neuroimaging techniques readily available, AVMs are more frequently diagnosed due to symptoms other than a hemorrhage. Headaches, seizures, and neurological deficits due to mass effect or steal phenomenon are among the most common. The growing availability of MRI has also led to an increase in the incidental detection of AVMs. In a recent analysis of incidental findings noted on brain MRI in 206 patients, cerebral AVMs were observed in 2 patients. ${ }^{6}$ Treatment for asymptomatic AVMs certainly requires rigid scrutiny, as the treatment is prophylactic and occasionally the complications of the treatment may be detrimental. In our series of 31 patients with incidental AVMs, 26 (84\%) obtained a complete obliteration of the nidi based on angiography or MRI following GKS. Only 2 patients $(6.5 \%)$ developed symptomatic radiationinduced imaging changes, with 1 patient experiencing temporary headache and 1 developing seizures and mild neurological deficits. Symptoms of both patients resolved with a short course of steroids. Two patients each had 1 hemorrhage during the latency period, while none of the patients with an obliterated nidus suffered a hemorrhage. There were no other acute or chronic procedure-related adverse events associated with this minimally invasive outpatient procedure. In addition to the favorable benefitto-risk profile afforded by GKS, the relief of psychological burdens in some patients who have difficulty coping with the idea of having a "low risk" intracranial vascular malformation might be significant, although difficult to objectively quantify.

With more risk-factor studies available, patient-specific or angioarchitectural factors have been tested to identify patients at higher risk of hemorrhage, and these patient- or AVM-related factors will likely dictate the initiation or selection of appropriate management modali- 
ties for unruptured or asymptomatic AVMs. Along with growing evidence of the benefits and complications associated with surgical, endovascular, and radiosurgical treatment for AVMs, we hope the long-term (i.e., at least 5 -year) outcome of ARUBA will provide more insightful information regarding the benefit-to-risk ratio of conservative management for unruptured AVMs. The present study, although limited by its small patient population and retrospective nature, offers the first reported imaging and clinical outcome of GKS for purely asymptomatic AVMs emphasizing the incidence and severity of complications associated with treatment, and can be eventually used as a comparison group for patients managed conservatively.

While this study does represent an experience derived of 20 years, there are limitations to its findings. Selection biases for referring patients and treating them may preclude generalization of these findings to all patients with asymptomatic AVMs. Also, the radiosurgical technique has become more refined over the study period. Finally, the statistical power of the study is small due to the size of the patient cohort.

\section{Conclusions}

Compared with embolization and microsurgical resection, radiosurgery is the least invasive treatment modality for AVMs. We report the first cohort of patients with unruptured, asymptomatic AVMs treated with GKS. The overall obliteration rate was high and increased in AVMs with smaller volumes, and the rate of postradiosurgical hemorrhage, symptomatic radiation adverse effects, and clinical deterioration was acceptably low. Eighty-four percent of patients with a total obliteration of their AVMs were devoid of future hemorrhage risk, at least during the 80-month follow-up period. However, it remains to be determined how these radiosurgical treatment outcomes compare with the natural history of AVMs pending the long-term results of randomized, prospective trials that include large patient cohorts. However, we remain cautiously optimistic that radiosurgery is a safe and effective therapy for at least some patients harboring incidental AVMs.

\section{Disclosure}

The authors report no conflict of interest concerning the materials or methods used in this study or the findings specified in this paper.

Author contributions to the study and manuscript preparation include the following. Conception and design: Yen. Acquisition of data: Yen, Cheng. Analysis and interpretation of data: Yen, Ding, Cheng. Drafting the article: Yen. Critically revising the article: all authors. Reviewed submitted version of manuscript: all authors. Approved the final version of the manuscript on behalf of all authors: Sheehan. Statistical analysis: Yen. Administrative/technical/material support: Sheehan, Shaffrey. Study supervision: Sheehan, Yen, Shaffrey.

\section{References}

1. Al-Shahi R, Bhattacharya JJ, Currie DG, Papanastassiou V, Ritchie V, Roberts RC, et al: Prospective, population-based detection of intracranial vascular malformations in adults: the Scottish Intracranial Vascular Malformation Study (SIVMS). Stroke 34:1163-1169, 2003

2. Brown RD Jr, Wiebers DO, Forbes G, O'Fallon WM, Piepgras DG, Marsh WR, et al: The natural history of unruptured intracranial arteriovenous malformations. J Neurosurg 68:352357, 1988

3. Crawford PM, West CR, Chadwick DW, Shaw MD: Arteriovenous malformations of the brain: natural history in unoperated patients. J Neurol Neurosurg Psychiatry 49:1-10, 1986

4. Ding D, Yen CP, Xu Z, Starke RM, Sheehan JP: Radiosurgery for patients with unruptured intracranial arteriovenous malformations. Clinical article. J Neurosurg 118:958-966, 2013

5. Fiehler J, Stapf C: ARUBA-beating natural history in unruptured brain AVMs by intervention. Neuroradiology 50:465467, 2008

6. Hartwigsen G, Siebner HR, Deuschl G, Jansen O, Ulmer S: Incidental findings are frequent in young healthy individuals undergoing magnetic resonance imaging in brain research imaging studies: a prospective single-center study. J Comput Assist Tomogr 34:596-600, 2010

7. Itoyama Y, Uemura S, Ushio Y, Kuratsu J, Nonaka N, Wada H, et al: Natural course of unoperated intracranial arteriovenous malformations: study of 50 cases. J Neurosurg 71:805-809, 1989

8. Karlsson B, Lax I, Söderman M: Risk for hemorrhage during the 2-year latency period following gamma knife radiosurgery for arteriovenous malformations. Int J Radiat Oncol Biol Phys 49: 1045-1051, 2001

9. Mathiesen T: Arguments against the proposed randomised trial (ARUBA). Neuroradiology 50:469-471, 2008

10. Ondra SL, Troupp H, George ED, Schwab K: The natural history of symptomatic arteriovenous malformations of the brain: a 24-year follow-up assessment. J Neurosurg 73:387391, 1990

11. Pollock BE, Flickinger JC: Modification of the radiosurgerybased arteriovenous malformation grading system. Neurosurgery 63:239-243, 2008

12. Yamane F, Takeshita M, Izawa M, Kagawa M, Sato K, Takakura K: Natural history of arteriovenous malformations: analysis of non-radically treated patients. J Clin Neurosci 5 Suppl:26-29, 1998

13. Yen CP, Cifarelli C, Schlesinger D, Sheehan JP: Stereotactic radiosurgery of vascular malformations, in Ellenbogen RG, Abdulrauf SI, Sekhar LN (eds): Principles of Neurological Surgery. Philadelphia: Elsevier, 2012, pp 715-725

14. Yen CP, Matsumoto JA, Wintermark M, Schwyzer L, Evans AJ, Jensen ME, et al: Radiation-induced imaging changes following Gamma Knife surgery for cerebral arteriovenous malformations. Clinical article. J Neurosurg 118:63-73, 2013

15. Yen CP, Sheehan JP, Schwyzer L, Schlesinger D: Hemorrhage risk of cerebral arteriovenous malformations before and during the latency period after GAMMA knife radiosurgery. Stroke 42:1691-1696, 2011

16. Yen CP, Steiner L: Gamma knife surgery for brainstem arteriovenous malformations. World Neurosurg 76:87-95, 57-58, 2011

Manuscript submitted July 2, 2013.

Accepted July 9, 2014.

Please include this information when citing this paper: published online August 22, 2014; DOI: 10.3171/2014.7.JNS131397. Address correspondence to: Jason Sheehan, M.D., Ph.D., Department of Neurological Surgery, University of Virginia, P.O. Box 800212, Charlottesville, VA 22908. email: jps2f@virginia.edu. 